\title{
BIOCRUDE PRODUCTION BY HYDROTHERMAL LIQUEFACTION OF OLIVE RESIDUE
}

\author{
P. DE FILIPPIS, B. DE CAPRARIIS, M. SCARSELLA, A. PETRULLO \& N. VERDONE \\ Chemical Engineering Department, Sapienza University of Rome, Italy.
}

\begin{abstract}
Hydrothermal liquefaction (HTL) converts biomass into a crude bio-oil by thermally and hydrolytically decomposing the biomacromolecules into smaller compounds. The crude bio-oil, or biocrude, is an energy dense product that can potentially be used as a substitute for petroleum crudes. Liquefaction also produces gases, solids, and water-soluble compounds that can be converted to obtain valuable chemical species or can be used as energy vectors. The process is usually performed in water at $250^{\circ} \mathrm{C}-370^{\circ} \mathrm{C}$ and under pressures of 4-22 MPa: depending on the adopted pressure and temperature the process can be carried out in sub-critical or super-critical conditions. In the conditions reached in hydrothermal reactors, water changes its properties and acts as a catalyst for the biomass decomposition reactions. One of the main advantages of this process is that the energy expensive biomass-drying step, required in all the thermochemical processes, is not necessary, allowing the use of biomass with high moisture content such as microalgae or olive residue and grape mark.

In this work, the feasibility of a hydrothermal process conducted under sub-critical conditions to obtain a bio-oil from the residue of olive oil production is investigated. The experimental tests were performed at $320^{\circ} \mathrm{C}$ and about $13 \mathrm{MPa}$, using a biomass to water weight ratio of 1:5. The influence of two different catalysts on the bio-oil yield and quality was investigated: $\mathrm{CaO}$ and a zeolite (faujasite$\mathrm{Na}$ ). $\mathrm{CaO}$ allows the increase of bio-oil yields, while the selected zeolite enhances the deoxygenation reactions, thus improving the bio-oil quality in terms of heating value.
\end{abstract}

Keywords: Hydrothermal liquefaction; biomass; olive residue;

\section{INTRODUCTION}

The increasing $\mathrm{CO}_{2}$ emissions have challenged the scientific community to find new techniques to exploit renewable energy in order to meet the world's growing needs for energy while reducing greenhouse gases. Nowadays, renewable energies represent less than $20 \%$ of the worldwide energy demand but this percentage is expected to increase at about $30 \%$ by 2035in the global energy supply [1]. Among renewables, biomass is an important feedstock for the production of fuels, chemicals and energy. Biomass, in fact, is a clean, cost-effective, $\mathrm{CO}_{2}$ neutral and low sulphur content renewable material and furthermore it is one of the largest sources of energy in the world. Biomass includes different kinds of biological matter: wood, agricultural waste, energy crops, algae, animal waste, organic fraction of municipal waste and others.

The main problem in using biomass as feedstock in energy conversion processes relies in its low energy density and high water content, which can reach up to $40 \%$ of the weight of the fuel, making it not convenient from an energetic point of view. Thus, it can be convenient to transform the solid biomass into more valuable fuels such as bio-oil and bio-syngas [2]. Traditional thermochemical processes such as pyrolysis and gasification present some weak 
points: the necessity of dry feedstocks to achieve a positive energy balance and of a further process to up-grade the raw product in order to obtain commercially valuable liquid and gaseous fuels.

Hydrothermal liquefaction (HTL) can be an interesting technology to produce a liquid biofuel from a wet biomass so without the need of the energy consuming drying process. In this process besides the bio-oil other products are formed: light gases, principally $\mathrm{CO}_{2}$ and small amount of $\mathrm{CH}_{4}, \mathrm{CO}$ and $\mathrm{H}_{2}$, a solid residue, char, and a water stream having high organic carbon content. In a HTL process, wet biomass and a supplementary amount of water if needed (biomass:water weight ratio is in general kept in the range 1:10-1:5), are heated in the range of $280^{\circ} \mathrm{C}-370^{\circ} \mathrm{C}$ under a pressure comprised between 10 and $25 \mathrm{MPa}$ [3, 4].At these pressures and temperatures, water is still in liquid phase and near its critical point that occurs at $374^{\circ} \mathrm{C}$ and $22.1 \mathrm{MPa}$. Water, in these conditions, assumes particular properties: the ionic product increases so that water can act as an effective medium for acid-base catalyzed organic reactions, while the viscosity decreases providing a higher diffusion coefficient that leads to higher global reaction rates [5]. Furthermore, at the high temperatures and pressures used in the HTL process water behaves as a reactant participating in the hydrolysis reaction as hydrogen donor needed to obtain a high quality bio-oil having lower oxygen content [3].

In this work, the $\mathrm{HTL}$ of olive residue is investigated at the temperature of $320^{\circ} \mathrm{C}$ and a pressure of about $13 \mathrm{MPa}$ and at biomass:water ratio of 1:5. The influence of two different catalysts on the bio-oil yield and quality was investigated: $\mathrm{CaO}$ and a zeolite (faujasite-Na). In literature, it is well-known that the addition of an alkali species has a positive effect on HTL increasing the liquid yield, but no mechanism for this catalytic action has been so far suggested [6]. The zeolite enhances the de-oxygenation reactions leading to a higher quality bio-oil [7]. To evaluate the feasibility of the process a qualitative techno-economic analysis was also conducted.

\section{EXPERIMENTAL SECTION}

\subsection{Experimental set-up}

The hydrothermal tests were carried out in a $300 \mathrm{~cm}^{3}$ batch stainless steel autoclave equipped with an overhead mechanical stirrer, a manometer, a K-Type thermocouple and a valve for the gas inlet-outlet. The experimental set-up is shown in Fig. 1. The autoclave was heated by an electrical heater to $320^{\circ} \mathrm{C}$ with continuous stirring and then maintained at this temperature for $30 \mathrm{~min}$. The pressure, continuously monitored during the process, was autogenous and its maximum was in the range of 13-14 MPa. The increase of temperature and consequentially of pressure of a HTL test are reported in Fig. 2. The catalysts used were $\mathrm{CaO}$ and a zeolite (faujasite-Na), both in an amount of $5 \%$ by weight with respect to the biomass.

In each liquefaction test, performed in non-catalytic and catalytic modality, $20 \mathrm{~g}$ of olive residue and $100 \mathrm{~g}$ of deionized water were loaded into the reactor, which was then sealed and purged with $\mathrm{N}_{2}$ for 3 min to remove residual air. After the reaction ended the autoclave was cooled down to room temperature by means of cooling coils installed inside the reactor. When the temperature reached $40^{\circ} \mathrm{C}$, the gas was vented off and analysed with a gas-chromatograph. Then the autoclave was opened and the reaction mixture was carefully collected for analysis according to the procedure described in the diagram depicted in Fig. 3. All runs under identical conditions were carried out in triplicate to confirm the repeatability of results. The reported values are average ones. 


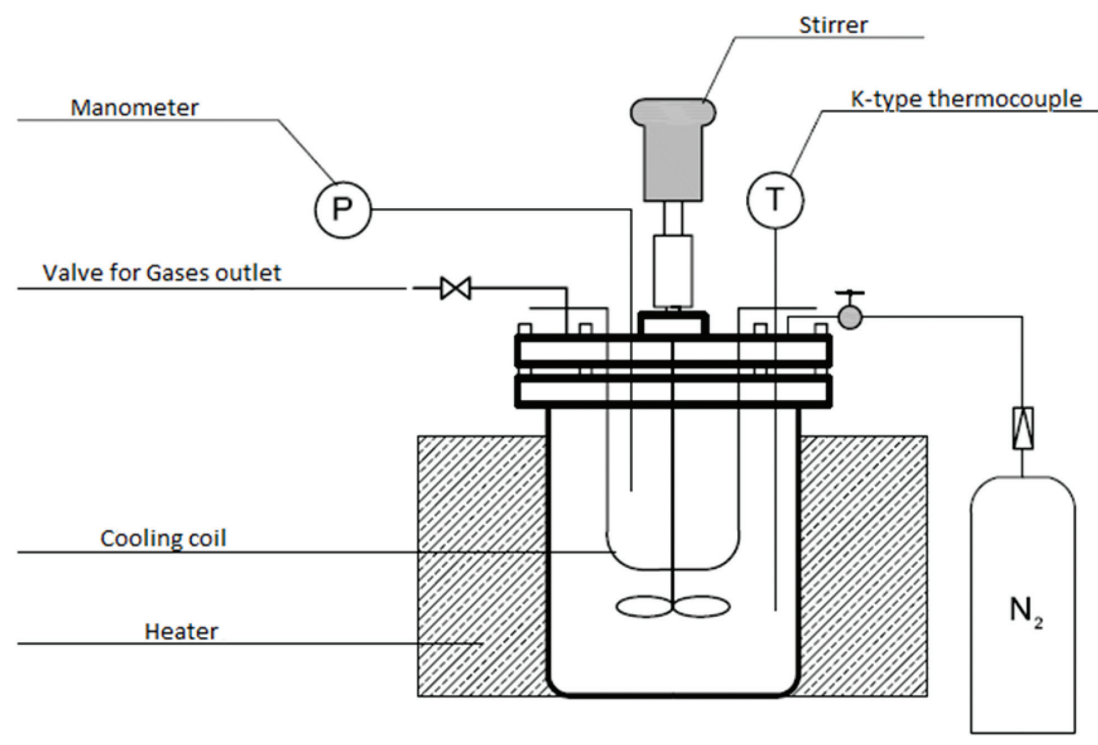

Figure 1: Experimental apparatus.

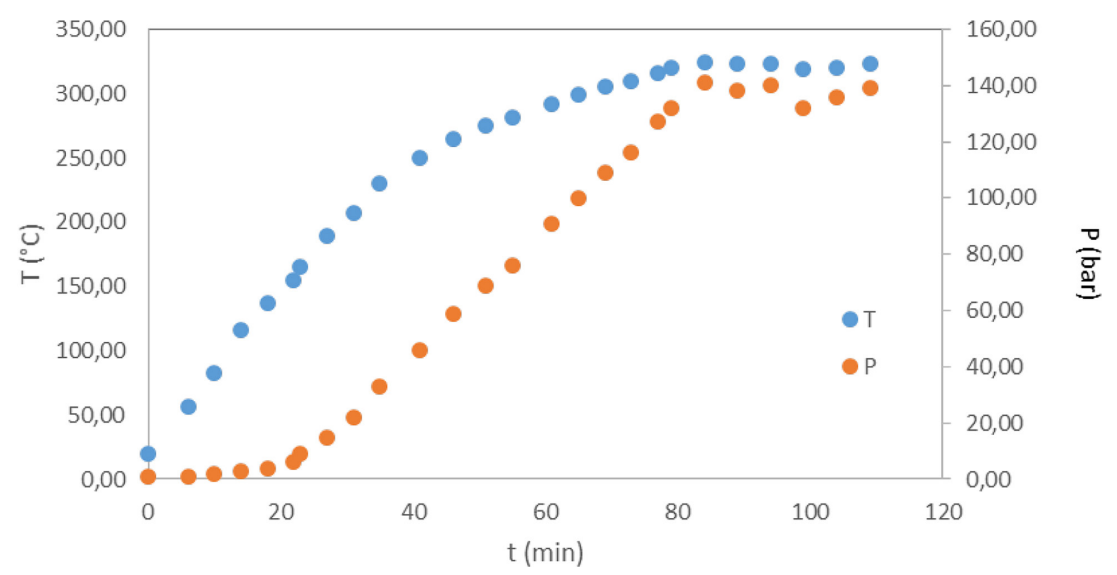

Figure 2: Temperature and pressure trends during HTL tests.

The reaction mixture was separated by filtration into a liquid aqueous phase containing the dissolved organic compounds and a solid product. The solid, on the filter paper, was washed with acetone in a Soxhlet extractor to recover oil-like compounds. The oil was obtained from the extract solution evaporating acetone at $60^{\circ} \mathrm{C}$ by using a rotary evaporator. The acetone insoluble fraction remaining on the filter was dried at $105^{\circ} \mathrm{C}$ for $24 \mathrm{~h}$ to yield the solid residue.

The organic soluble fraction in the water phase was measured with a Total Organic Carbon Analyzer (TOC-L Shimadzu).

The oil fraction was analysed with a CHNS Elemental Analyser (Eurovector) to determine its elemental composition and with a Gas Chromatography-Mass Spectrometry instrument to determine the organic compounds constituting it.

The elemental composition of the solid residue was also analysed. 


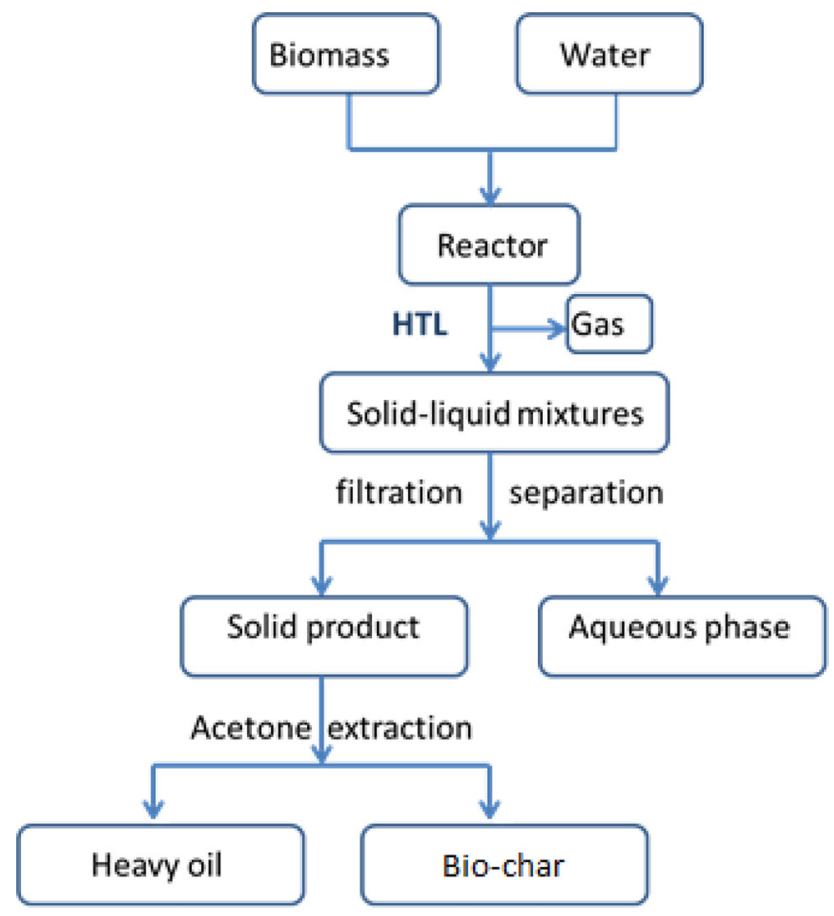

Figure 3: Procedures for separation and extraction of HTLproducts.

\subsection{Materials}

The physical and chemical properties of the olive residue are reported in Table 1.

\section{RESULTS AND DISCUSSION}

\subsection{Hydrothermal products characterization}

The distribution of the three products obtained during the process is reported in Table 2. It can be noticed that the oil fraction increases with the use of the alkaline catalyst while slightly decreases with the zeolite. This behaviour was expected since, as already said, the alkalicatalysed process leads to higher amount of bio-oil. The minor amount of oil obtained using the zeolite can be attributed to its deoxygenation action. In fact the deoxygenation reactions lead to the formation of $\mathrm{CO}_{2}$ and $\mathrm{H}_{2} \mathrm{O}$ causing an aromatization of the oil compounds $(\mathrm{H} / \mathrm{C}$ ratio decreases), a decrease of the oil amount and furthermore a double quantity of gas recovered [8]. These results are confirmed by the analysis of the elemental composition of the three bio-oils reported in Table 3. The zeolite process produces a bio-oil with the lowest $\mathrm{O} / \mathrm{C}$ ratio and thus with a higher quality. The oxygen causes instability of the bio-oil, leading to problems during the storage phase. The oxygenated species, indeed, are very reactive, particularly aldehydes and ketones, causing transformations of the oil composition and properties. For example, the oil viscosity significantly increases after a short period during the oil storage. 
Table 1: Physical and chemical properties of olive residue.

Proximate analysis

Moisture (\%)

$10-20$

Volatile substance (\% dry basis)

65

Fixed carbon (\% dry basis)

29.6

Ash (\% dry basis)

5.4

Size (mm)

$<1.0$

High heating value $(\mathrm{MJ} / \mathrm{kg})$

17.6

Elemental analysis (\% dry basis)

Carbon

44.2

Hydrogen

5.8

Nitrogen

1.8

Oxygen

48.2

Sulphur

0

Table 2: HTL products.

\begin{tabular}{lcccc}
\hline & $\begin{array}{c}\text { Oil fraction } \\
(\%)\end{array}$ & $\begin{array}{c}\text { Char } \\
(\%)\end{array}$ & $\begin{array}{c}\text { Gas } \\
(\%)\end{array}$ & $\begin{array}{c}\mathrm{TOC} \mathrm{ofH}_{2} \mathrm{O} \\
(\mathrm{ppm})\end{array}$ \\
\hline No catalyst & 32.40 & 19.60 & 7.80 & 21,300 \\
$\mathrm{CaO}$ & 38.05 & 18.65 & 5.65 & 23,400 \\
Zeolite & 29.10 & 19.90 & 14.90 & 25,230 \\
\hline
\end{tabular}

Table 3: Oil elemental composition and calorific value.

\begin{tabular}{lccccccc}
\hline & $\mathrm{C}$ & $\mathrm{H}$ & $\mathrm{N}$ & $\mathrm{O}$ & $\mathrm{O} / \mathrm{C}$ & $\mathrm{H} / \mathrm{C}$ & $\mathrm{HHV}(\mathrm{MJ} / \mathrm{kg})$ \\
\hline No catalyst & 63.27 & 7.88 & 0.73 & 28.12 & 0.44 & 0.125 & 28.71 \\
$\mathrm{CaO}$ & 64.48 & 8.67 & 1.64 & 25.21 & 0.39 & 0.134 & 30.69 \\
Zeolite & 66.67 & 7.94 & 1.46 & 23.93 & 0.36 & 0.119 & 30.49 \\
\hline
\end{tabular}




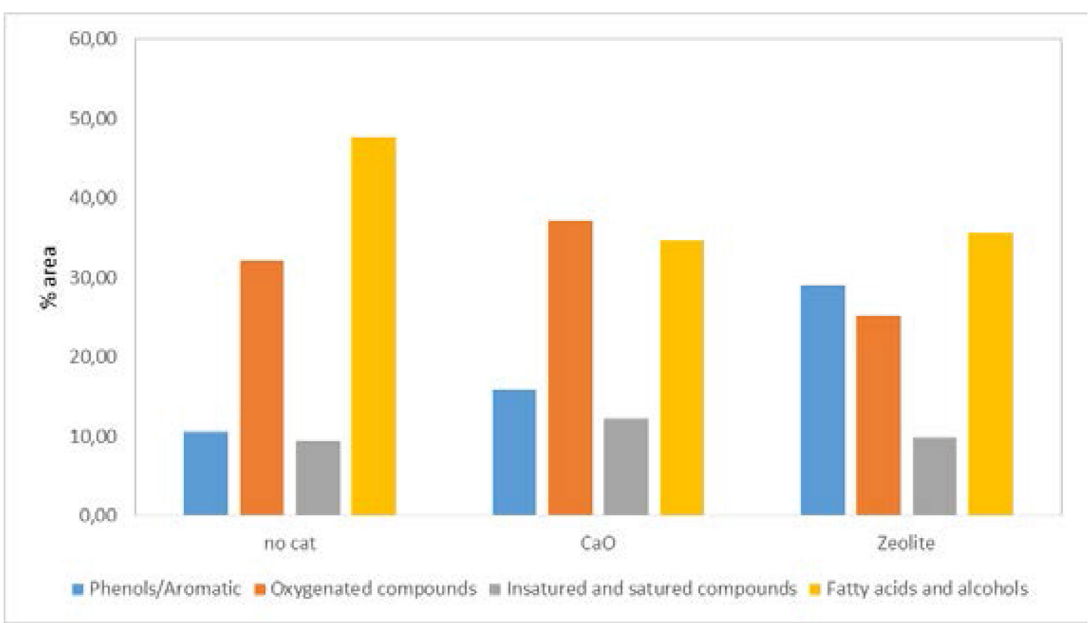

Figure 4: Bio-oil composition for the three obtained samples.

Compared with traditional pyrolysis, the bio-oils obtained with HTL present a higher quality having consistently minor oxygen content than the average oxygen content (35\%-40\%) of a traditional pyrolysis oil [9].

Larger amount of bio-oil could be obtained extracting part of the organic compounds dissolved in water. In fact, from the measured TOC values it can be argued that about $20 \%$ of the biomass carbon is recovered in the water soluble species.

To summarize the differences in bio-oil composition depending on the type of catalyst used, in Fig. 4 the relative abundance of the main chemical classes of the oily fraction is reported. Phenols and aromatic compounds formed during the decomposition of the lignin fraction of the biomass range between 10 and 30\% of the totality of the chemical organic compounds. The $30 \%$ is reached when the zeolite catalyst is used, favouring the aromatization of the molecules. The bio-oil is also rich of long chain fatty acids obtained principally from the decomposition of the lipid fraction of the biomass, whose amount is high in the olive residue (about 10\%) [10].

\subsection{Energy analysis}

To evaluate the feasibility of the process from an energetic point of view, two indexes were calculated, the energy recovery (ER) and the energy consumption ratio (ECR), whose values were compared with those obtained in traditional fixed-bed pyrolysis process [9].

The ER measures the ratio between the high calorific value of the product and the high calorific value of the feedstock. The higher this index the most convenient is the process.

The ECR is the ratio between the energy demand for the HTL process and the energy available in the bio-oil (eqn(1)), where ECR values less than unity indicate a positive energy balance.

$$
E C R=\frac{\left[W_{i} C_{p w} T+\left(1-W_{i}\right) C_{p b} T\right] *\left(1-R_{h}\right)}{Y * H H V^{*}\left(1-W_{i}\right) * R_{C}}
$$


Table 4: ER and ECR indexes for the HTL tests.

\begin{tabular}{llc}
\hline & ER & ECR \\
\hline HTL (no cat) & 53.0 & 0.41 \\
HTL (CaO) & 66.3 & 0.32 \\
HTL (zeolite) & 50.4 & 0.42 \\
Pyrolysis & 26.0 & - \\
\hline
\end{tabular}

where $W_{i}$ is the initial water fraction with respect to the biomass, $T$ is the temperature increase required to reach conversion conditions (initial temperature assumed to be $25^{\circ} \mathrm{C}$ ), $C_{p w}$ and $C_{p b}$ are the specific heats of water and biomass, $R_{h}$ and $R_{c}$ are the efficiencies of heat recovery and combustion energy assumed to be 0.5 and 0.7 , respectively, $Y$ is the bio-oil fraction, and $H H V$ is the higher heating value of the bio-oil.

The values of the ER and ECR, reported in Table 4, indicate that there is always a positive energy balance. The process with $\mathrm{CaO}$ catalyst presents the highest convenience due to the larger amount of bio-oil produced. With respect to the traditional pyrolysis process, the benefit in terms of energy balance of the HTLis evident.

\section{CONCLUSIONS}

In this work, a HTL process to produce bio-oil from olive residue was investigated. The use of two different catalysts was tested to obtain a bio-oil with improved yield and quality.

The bio-oil yields range between $29 \%$ and $38 \%$; the maximum amount of oil is obtained using $\mathrm{CaO}$ as catalyst and the minimum using a Na-zeolite. The analyses of the oil composition show that zeolite allows increasing the oil quality at the expense of the quantity due to the occurrence of deoxygenation reactions; the $\mathrm{O} / \mathrm{C}$ ratio, indeed, decreases from 0.44 to 0.36. These values are much lower with respect to those obtained for bio-oil produced by traditional pyrolysis $(\mathrm{O} / \mathrm{C}$ ratio about 0.7$)$.

The energy analysis indicates that there is always a positive energy balance especially in the case of reaction catalyzed by $\mathrm{CaO}$ due to the higher oil yield.

The results demonstrate that HTL can be an attractive alternative to traditional thermal treatments to produce biocrude having quality closer to that of fossil fuels with respect to traditional bio-oil.

\section{REFERENCES}

[1] Lee, J.W., Hawkins, B., Day, D.M. \& Reicosky, D.C. Sustainability: the capacity of smokeless biomass pyrolysis for energy production, global carbon capture and sequestration. Energy Environment Science, 3, pp. 1695-1705, 2010. http://dx.doi.org/10.1039/c004561f

[2] Bassano, C. \& Deiana, P. Carbon dioxide reforming of tar during biomass gasification. Chemical Engineering Transaction, 37, pp. 97-102, 2014. 
[3] De Filippis, P., de Caprariis, B., Scarsella, M. \& Verdone, N. The hydrothermal decomposition of biomass and waste to produce bio-oil. WIT Transactions on Ecology and the Environment, 180, pp. 445-451, 2014. http://dx.doi.org/10.2495/WM140381

[4] Haarlemmer, G.,Guizani, C., Anouti, S., Deniel, M., Roubaud, A. \& Valin, S. Analysis and comparison of bio-oils obtained by hydrothermal liquefaction and fast pyrolysis of beech wood. Fuel, 174, pp. 180-188, 2016. http://dx.doi.org/10.1016/j.fuel.2016.01.082

[5] Déniel, M., Haarlemmer, G., Roubaud, A., Weiss-Hortala, E. \& Fages, J. Energy valorisation of food processing residues and model compounds by hydrothermal liquefaction. Renewable and Sustainable Energy Reviews, 54, pp. 1632-1652, 2016. http://dx.doi.org/10.1016/j.rser.2015.10.017

[6] Tekin, K., Karagöz, S. \& Bektaş, S. A review of hydrothermal biomass processing. Renewable and Sustainable Energy Reviews, 40, pp. 673-687, 2014. http://dx.doi.org/10.1016/j.rser.2014.07.216

[7] Mortensena, P.M., Grunwaldt, J.D., Jensena, P.A., Knudsenc, K.G. \& Jensen A.D. A review of catalytic upgrading of bio-oil to engine fuels. Applied Catalysis A: General, 407, pp.1-19, 2011. http://dx.doi.org/10.1016/j.apcata.2011.08.046

[8] Mohan, D., Pittman, C.U. \& Steele, P.H. Pyrolysis of wood/biomass for bio-oil: acritical review. Energy \& Fuels, 20, pp. 848-889, 2006. http://dx.doi.org/10.1021/ef0502397

[9] Vardon, D.R., Sharma, B.K., Blazina, G.V., Rajagopalan, K. \& Strathmann, T.J. Thermochemical conversion of raw and defatted algal biomass via hydrothermal liquefaction and slow pyrolysis. Bioresource Technology, 109, pp. 178-187, 2012. http://dx.doi.org/10.1016/j.biortech.2012.01.008

[10] de Caprariis, B.,Santarelli, M.L., Scarsella, M., Herce, C., Verdone, N. \& De Filippis, P. Kinetic analysis of biomass pyrolysis using a double distributed activation energy model. Journal of Thermal Analysis and Calorimetry, 121(3), pp. 1403-1410, 2015. http://dx.doi.org/10.1007/s10973-015-4665-2 